\title{
Presencia de haplotipos reproductivos y su efecto en una población de vacunos lecheros en la costa central del Perú
}

\author{
Presence of reproductive haplotypes and their effect on a dairy cattle population in \\ the central coast of Peru
}

\author{
Jorge Pedro Calderón Velásquez ${ }^{1,3}$, Gustavo Augusto Gutiérrez Reynoso ${ }^{1}$, Gladys \\ Luz Garay Livia ${ }^{2}$
}

\section{Resumen}

\begin{abstract}
Se utilizaron 6350 registros de reproducción de un rebaño lechero entre 2000 hasta el primer semestre de 2016, con base a los índices reproductivos del rebaño, considerando el número de servicios por preñez, días vacíos, tasa de preñez y ocurrencia de abortos. Se genotiparon 58 vacas Holstein seleccionadas al azar para determinar la presencia de haplotipos reproductivos $(\mathrm{HH})$ que afectan el comportamiento reproductivo de las vacas produciendo abortos. Se determinó que el 5.2\% de las vacas eran portadoras para $\mathrm{HH} 1,5.2 \%$ para $\mathrm{HH} 3$ y $3.4 \%$ para $\mathrm{HH}$, no encontrándose animales portadores para los haplotipos HH2 y HH4. El 75\% de las vacas portadoras (6/8) presentaron abortos no asociados al haplotipo, a excepción de un evento cuyo reproductor fue portador para el HH3. Asimismo, el 54\% de las vacas no portadoras presentaron eventos de aborto $(27 / 50)$, no estableciéndose un efecto directo del haplotipo sobre la ocurrencia de abortos. El número de servicios por preñez asociado con la tasa de concepción genómica (CCR) determinó una correlación de $8.3 \%(\mathrm{p}>0.05)$ y un coeficiente de regresión de 0.07115 servicios $(\mathrm{p}>0.05$ ). De los cinco grupos genéticos de padres encontrados en el rebaño, cuatro grupos genéticos tienen presencia a través de la progenie de machos que presentaron los haplotipos reproductivos.
\end{abstract}

Palabra clave: haplotipos reproductivos, tasa de concepción, Holstein

\footnotetext{
${ }^{1}$ Departamento Académico de Producción Animal, Facultad de Zootecnia, Universidad Nacional Agraria La Molina, Lima, Perú

${ }^{2}$ Departamento Académico de Ingeniería, Escuela Profesional de Ingeniería Agrónoma, Universidad Nacional de Barranca, Lima, Perú

${ }^{3}$ E-mail: jcalderonv@lamolina.edu.pe; https://orcid.org/0000-0002-7545-3579
}

Recibido: 29 de mayo de 2019

Aceptado para publicación: 27 de febrero de 2021

Publicado: 23 de junio de 2021

CLos autores. Este artículo es publicado por la Rev Inv Vet Perú de la Facultad de Medicina Veterinaria, Universidad Nacional Mayor de San Marcos. Este es un artículo de acceso abierto, distribuido bajo los términos de la licencia Creative Commons Atribución 4.0 Internacional (CC BY 4.0) [https:// creativecommons.org/licenses/by/4.0/deed.es] que permite el uso, distribución y reproducción en cualquier medio, siempre que la obra original sea debidamente citada de su fuente original 
Reproductive records $(n=6350)$ of a dairy herd between 2000 and the first semester of 2016 were used based on the reproductive indices of the herd, considering the number of services per pregnancy, open days, pregnancy rate and occurrence of abortions. In total, 58 Holstein cows selected at random were genotyped to determine the presence of reproductive haplotypes $(\mathrm{HH})$ that affect the reproductive performance of cows causing abortions. It was determined that $5.2 \%$ of the cows were carriers for $\mathrm{HH} 1,5.2 \%$ for $\mathrm{HH} 3$ and 3.4\% for $\mathrm{HH} 5$, not finding carrier animals for the $\mathrm{HH} 2$ and $\mathrm{HH} 4$ haplotypes. Besides, $75 \%$ of the carrier cows (6/8) presented abortions not associated with the haplotype, except for one event whose reproducer was a carrier for HH3. Likewise, 54\% of the noncarrier cows presented abortion events (27/50), not establishing a direct effect of the haplotype on the occurrence of abortions. The number of services per pregnancy associated with the genomic conception rate (CCR) determined a correlation of $8.3 \%$ $(p>0.05)$ and a regression coefficient of -0.07115 services $(p>0.05)$. Of the five genetic groups of sire parenthood found in the herd, four genetic groups are present through the progeny of males that presented the reproductive haplotypes.

Key words: haplotypes reproductive, conception rate, Holstein Frisian

\section{INTRODUCCIÓN}

La ocurrencia de abortos o absorciones embrionarias es una de las principales causas de la baja eficiencia reproductiva en el ganado bovino (Disking y Morris, 2008; Disking et al., 2012). Estos pueden deberse a la depresión endogámica, observada por el incremento de la consanguinidad en los animales, principalmente en el ganado bovino, $\mathrm{o}$ a efectos genéticos (Mylrea, 1963; Wijeratne y Stewart, 1971), determinado por la presencia de haplotipos asociados a la reproducción en estado homocigota del embrión (VanRaden et al., 2012).

Diversas investigaciones han identificado la presencia de haplotipos relacionados con la reducción de las tasas reproductivas. Al inicio se reportaron tres haplotipos (VanRaden et al., 2011) y hacia 2017 se sumaron dos más para el ganado Holstein (Cooper et al., 2013), los cuales se asocian a efectos negativos en el desarrollo embrionario y abortos (Norman et al., 2012).
Los efectos negativos de la presencia de estos haplotipos en la reproducción estiman un impacto sobre la tasa de concepción de $-0.35 \%$ para el tipo $\mathrm{HH} 1$, y algo similar $(-0.36 \%)$ para el haplotipo $\mathrm{HH} 2$ y HH3, y cuyas frecuencias de los heterocigotos fueron reportadas de 4.5, 4.6 y $4.7 \%$ respectivamente (VanRaden et al., 2011). Al evaluar la tasa de no retorno a 60 días (NR60) se estimó un impacto fue de $-1.1,-1.7$ y -3.1\% para los haplotipos HH1, HH2 y HH3, respectivamente. Por otro lado, Fritz et al. (2013) reportaron frecuencias de 3.6 y $3.9 \%$ para los haplotipos $\mathrm{HH} 4$ y HH5, respectivamente, asociado a una pérdida en nacimientos en vacas en $-1.74 \%$ para el $\mathrm{HH} 4$ y de $-2.17 \%$ para el HH5.

Asimismo, los haplotipos han sido relacionados a grupos genéticos de toros que fueron utilizados en poblaciones de vacunos Holstein, tanto para obtener machos reproductores como vacas productoras, lo cual generó un impacto en la incidencia y presencia de los haplotipos reproductivos en la ganadería lechera local. Reproductores tales 
como Pawnee Farm Arlinda CHIEF y Walkway Chief MARK, en quienes se reporta la presencia del haplotipo HH1 (Larkin et al., 2012, VanRaden et al., 2012), han sido utilizados para producir progenie orientada a seleccionar machos para su empleo en centrales de inseminación artificial, generando una gran influencia genética en la población de vacas resultantes.

El objetivo de la presente investigación fue determinar, en un rebaño lechero en la costa del Perú, la presencia de haplotipos reproductivos asociados con una baja eficiencia reproductiva, así como determinar la correlación entre la tasa de concepción genómica en vacas y el número de servicios por preñez.

\section{Materiales y Métodos}

Los datos del estudio fueron obtenidos de la base de datos de una ganadería bajo un sistema intensivo dedicada a la crianza de vacunos lecheros en la costa central del Perú. Los animales de fundación correspondieron a genética norteamericana. De los 8833 registros de producción/ reproducción con que cuenta la empresa, se consideraron 6350 registros que corresponden a los años 20002016 (primer semestre), de 2628 vacas en total, con una media de los últimos cinco años de 509 vacas por año. El establo se encuentra certificado libre de TBC y brucelosis por el Servicio Nacional de Sanidad Agraria (SENASA).

El sistema de manejo de la ganadería se basa en un sistema de alimentación TMR (Ración Totalmente Mezclada), determinándose seis grupos de alimentación: vacas recién paridas, de alta, media y baja producción, vacas en seca y preparto. La detección de celo se realizó mediante el uso de podómetros Westfalia GEA ${ }^{\mathrm{TM}}$, que es un sistema de medición de actividad y monitoreado por el software de gestión de rebaños Dairy Plan C21 $1^{\mathrm{TM}}$. Para la confirmación de preñez a los 35 días se utilizó un ecógrafo portátil (Caresono SD9000).

Los abortos registrados por año, el perfil reproductivo del rebaño medido como el promedio de servicios por preñez, el promedio de días vacíos, y la tasa de preñez fue calculado siguiendo la metodología descrita por VanRaden et al. (2004) y se presenta en el Cuadro 1. En el periodo 2000-2016 re registró 422 casos de natimortos y 426 abortos (Cuadro 2).

Se construyó la genealogía con base a la información de los registros genealógicos, tanto de la línea paterna como materna, para ser analizados con los programas informáticos Endog V4.8 (Gutiérrez y Goyache, 2005) y Pedigraph V2.4 (Garbe y Da, 2008), identificando 17478 animales, utilizando la base de datos de la Asociación Holstein de los Estados Unidos (Holstein Association USA, 2017) y los certificados genealógicos del establo lechero en estudio.

El tamaño de la muestra a genotipar se determinó utilizando la metodología descrita por Krejcie y Morgan (1970), considerando una precisión de 0.05 , un tamaño de rebaño de 500 vacas, y la probabilidad de ocurrencia promedio de los haplotipos, obteniendo un tamaño de muestra de 59 animales. Se seleccionaron 60 animales para el genotipado utilizando una rutina para generar números aleatorios en una hoja electrónica usando la función aleatorio (), independiente si las vacas presentaban historial de abortos o un número determinado de partos.

Se tomaron muestras de pelo de la cola de los animales seleccionados, considerando un mínimo de 40 pelos con bulbo piloso intacto para la extracción de ADN. Las muestras se colocaron en las tarjetas colectoras otorgadas por el laboratorio y fueron enviadas al laboratorio de GeneSeek (Neogen Corpora- 
Cuadro 1. Distribución del número de abortos, número de servicios por preñez, días vacíos y tasa de preñez por año (periodo 2000-2016)

\begin{tabular}{cccccc}
\hline & \multicolumn{2}{c}{ Número de } & \multicolumn{2}{c}{ Promedio de } & Tasa de \\
\cline { 2 - 4 } Año & Vacas & Abortos & $\begin{array}{c}\text { Servicios/ } \\
\text { preñez }\end{array}$ & Días vacíos & preñez $(\%)$ \\
\hline 2000 & 396 & 14 & 2.67 & 163.8 & 18.3 \\
2001 & 354 & 12 & 2.66 & 152.7 & 20.2 \\
2002 & 332 & 12 & 2.74 & 162.6 & 18.5 \\
2003 & 304 & 25 & 3.00 & 166.5 & 17.9 \\
2004 & 301 & 28 & 2.68 & 192.2 & 14.7 \\
2005 & 243 & 11 & 2.55 & 189.0 & 15.0 \\
2006 & 253 & 26 & 2.54 & 196.3 & 14.2 \\
2007 & 298 & 17 & 2.97 & 226.1 & 11.9 \\
2008 & 344 & 15 & 2.90 & 187.8 & 15.1 \\
2009 & 346 & 17 & 2.90 & 183.9 & 15.6 \\
2010 & 417 & 19 & 3.21 & 225.0 & 11.9 \\
2011 & 435 & 16 & 3.08 & 213.9 & 12.7 \\
2012 & 476 & 23 & 4.29 & 246.6 & 10.6 \\
2013 & 487 & 29 & 3.92 & 224.3 & 12.0 \\
2014 & 541 & 87 & 4.61 & 218.1 & 12.4 \\
2015 & 607 & 53 & 4.19 & 212.5 & 12.8 \\
2016 & 216 & 22 & 3.94 & 193.1 & 14.6 \\
\hline Total & 6350 & 426 & 3.22 & 198.4 & 14.1 \\
\hline
\end{tabular}

tion, USA), siguiendo las regulaciones del Departamento de Agricultura de Estados Unidos de Norteamérica - Servicio de Inspección Sanitaria de Animales y Plantas (USDA APHIS) para muestras biológicas. El genotipado se realizó con el GeneSeek Genomic Profiler for Dairy-LD (GGP 50k, Igenity Prime). El análisis reportó resultados de 45 características que el USDA considera en sus evaluaciones genéticas y genómicas para ganado lechero Holstein, en la que incluye los haplotipos reproductivos y los valores de la tasa de concepción de la vaca genómico (CCR: Cow Conception Rate).
Cuadro 2, Natimortos y abortos, según el número de parto (2000-2016)

\begin{tabular}{ccc}
\hline Parto & Natimortos & Abortos \\
\hline 1 & 207 & 156 \\
2 & 85 & 117 \\
3 & 55 & 83 \\
4 & 38 & 34 \\
5 & 23 & 24 \\
$\geq 6$ & 14 & 12 \\
\hline Total & 422 & 426 \\
\hline
\end{tabular}


Cuadro 3. Frecuencias de haplotipos reproductivos en bovinos lecheros de una ganadería de la costa central del Perú

\begin{tabular}{ccc}
\hline Haplotipo & $\begin{array}{c}\text { Animales } \\
\text { heterocigotos } \\
(\mathrm{n})\end{array}$ & $\begin{array}{c}\text { Frecuen- } \\
\text { cia (\%) }\end{array}$ \\
\hline HH1 & 3 & 5.2 \\
HH3 & 3 & 5.2 \\
HH5 & 2 & 3.4 \\
\hline
\end{tabular}

El CCR es definido como la capacidad de quedar preñada una vaca en cada servicio, donde una puntuación de +1 indica que la vaca tiene un $1 \%$ más de probabilidades de quedar preñada que las vacas con una evaluación de 0 . La estimación de dicho valor genómico es determinada por la frecuencia alélica de la población base menos el genotipo y el efecto poligénico, con un 10\% de varianza aditiva y se ajusta el modelo: $\mathrm{DP}=$ media $+\mathrm{S}$ genotipos(efectos) + poli + error (CDCB, 2017).

\section{Resultados y Discusión}

De las 60 muestras enviadas a GeneSeek, solo se genotiparon 58, excluyéndose dos muestras por posible contaminación del ADN. Con los resultados del genotipado se determinaron las frecuencias de los portadores para los haplotipos asociados a la reproducción $(\mathrm{HH})$ y el valor genómico de la Tasa de concepción de las vacas (CCR), que es un indicador de la eficiencia reproductiva del punto de vista genómico.

\section{Haplotipos HH}

En los 58 genotipados se encontraron tres de los cinco haplotipos reportados en la- raza Holstein que se encuentran asociados a la baja eficiencia reproductiva (VanRaden et al., 2011; Cole et al., 2014). Estos fueron los haplotipos HH1, HH3 y HH5, cuyas frecuencias de portadoras fueron similares a las reportados en otras poblaciones de ganado Holstein (VanRaden et al., 2011, 2012; Fritz et al., 2013). Las frecuencias se presentan en el Cuadro 3.

Al analizar el comportamiento reproductivo de las vacas portadoras del haplotipo a través de los partos que han tenido, se encontró que el $75 \%$ de las vacas portadoras (6 de 8 vacas) presentaron abortos durante su vida productiva, independientemente del número de partos, como es el caso de la vaca 2781 que en tres de los cinco partos registrados presentó abortos. Se entiende que la presentación de aborto debe darse como causa del haplotipo en estado de homocigosis del embrión, que en el caso de la vaca 5191 pudo deberse ya que el embrión tenía una probabilidad de $25 \%$ de ser homocigota al ser el padre portador para $\mathrm{HH} 3$, mientras que en los otros casos los reproductores utilizados en la reproducción no fueron portadores para los haplotipos. La presentación de los sucesos de aborto se resume en el Cuadro 4.

El $54 \%$ de las vacas no portadoras (27/50) para los haplotipos reproductivos presentaron abortos a lo largo de su vida reproductiva, y el $46 \%$ restante no presentó abortos. En el análisis de los datos se observó que los toros utilizados en la inseminación artificial en el grupo de vacas que presentaron abortos fueron heterocigotos para los haplotipos reproductivos (Cuadro 5).

En el grupo de vacas portadoras, la media de gestación al aborto fue de 129.2 días, y para el grupo de vacas no portadoras que presentaron un evento de aborto fue de 134.7 días, valores muy similares, lo cual no permite establecer un efecto en la pérdida de la gestación atribuible a la presencia de los haplotipos reproductivos. 
Cuadro 4. Comportamiento reproductivo de las vacas heterocigotas para los haplotipos reproductivos

\begin{tabular}{|c|c|c|c|c|c|c|c|}
\hline Animal & Haplotipo & CCR & $\begin{array}{l}\text { Servicios } \\
\text { promedio } \\
\text { (n) }\end{array}$ & $\begin{array}{l}\text { Partos } \\
\text { (n) }\end{array}$ & $\begin{array}{l}\text { Número } \\
\text { de } \\
\text { gestación }\end{array}$ & $\begin{array}{l}\text { Días de } \\
\text { gestación }\end{array}$ & Observaciones \\
\hline 5041 & $\mathrm{HH} 1 \mathrm{C}$ & 0.70 & 3.00 & 2 & 2 & 275 & Aborto $167 \mathrm{~d}$. \\
\hline 5191 & $\mathrm{HH} 3 \mathrm{C}$ & 1.40 & 3.50 & 1 & 1 & 274 & $\begin{array}{l}\text { Aborto } 171 \\
d^{* *}\end{array}$ \\
\hline 5163 & HH5C & -1.00 & 2.00 & 1 & 1 & 272 & Aborto $251 \mathrm{~d}$ \\
\hline 2781 & HН3C & -0.20 & 3.70 & 5 & $2,3,5$ & 278 & $\begin{array}{l}\text { Aborto 79, 77, } \\
179 \mathrm{~d}\end{array}$ \\
\hline 2662 & $\mathrm{HH} 1 \mathrm{C}$ & -0.30 & 4.80 & 4 & $4 *$ & 276 & $\begin{array}{l}\text { Aborto 80, } 77 \\
\text { d }\end{array}$ \\
\hline 5000 & HH5C & 1.40 & 5.50 & 2 & 2 & 279 & Aborto $82 \mathrm{~d}$ \\
\hline 2809 & НH3C & 1.90 & 1.50 & 4 & & 277 & Normal \\
\hline 2886 & HH1C & 2.40 & 2.50 & 2 & & 274 & Normal \\
\hline Promedio & & 0.79 & 3.39 & 2.63 & & 276 & 9 abortos \\
\hline
\end{tabular}

\section{Tasa de Concepción de las vacas}

La tasa de concepción en el periodo de estudio fue menor en los últimos años que en los primeros años (Cuadro 6 ), pudiendo deberse a una posible endogamia del rebaño (Parland et al., 2007; González-Recio et al., 2007).

La Figura 1 muestra la reducción de la tasa de concepción por número de servicio entre los cuatro primeros años (2000-2003) y los cuatro últimos años (2013-2016), observándose una reducción significativa de la tasa de concepción (Cuadro 1).

Se encontró una baja correlación $(8.30 \%)$ entre los valores de la tasa de concepción determinados genómicamente (CCR) con el número de servicios efectivos para preñez $(p>0.05)$, y la tendencia de la varia- ción, calculado mediante el coeficiente de regresión, fue de -0.07115 servicios por valor de +1 de CCR, no siendo estadísticamente significativo, lo que permite indicar que la eficiencia de la reproducción, medido como CCR no es un buen indicador de la performance reproductiva de las vacas, ya que existen otros factores que pueden estar influenciando la tasa de preñez (Mylrea, 1963; Wijeratne y Stewart, 1971) (Ver Figura 2).

\section{Grupos Genéticos}

Al evaluar la información de la genealogía de las vacas se pudo establecer cinco grupos genéticos de padres (Cuadro 7), considerando como padre fundador aquel animal donde se reporta por primera vez el haplotipo o es ancestro común con los reproductores utilizados en la reproducción y, que, en muchos casos, se utilizaron reproductores portadores de los haplotipos tipo $\mathrm{HH}$. 
Cuadro 5. Comportamiento reproductivo de vacas no portadoras para los haplotipos reproductivos y que presentaron abortos

\begin{tabular}{|c|c|c|c|c|c|c|c|}
\hline Animal & Haplotipo & $\mathrm{CCR}$ & $\begin{array}{l}\text { Servicios } \\
\text { (n) }\end{array}$ & $\begin{array}{l}\mathrm{N}{ }^{\circ} \text { de } \\
\text { partos }\end{array}$ & $\begin{array}{c}\text { N. } .^{\circ} \text { de } \\
\text { gestación }\end{array}$ & $\begin{array}{l}\text { Largo de } \\
\text { gestación }\end{array}$ & Observaciones \\
\hline 4749 & $\mathrm{~T}$ & -0.50 & 2.90 & 3 & $2 *, 3$ & 276 & $\begin{array}{l}\text { Aborto 101, 88, } \\
195 \text { d }\end{array}$ \\
\hline 5145 & $\mathrm{~T}$ & 3.60 & 2.80 & 4 & 2 & 271 & Aborto $103 \mathrm{~d}$ \\
\hline 2801 & $\mathrm{~T}$ & -1.00 & 5.25 & 3 & 2 & 275 & Aborto $111 \mathrm{~d}$ \\
\hline 2762 & $\mathrm{~T}$ & 2.00 & 3.00 & 6 & 2,6 & 274 & Aborto $113,125 \mathrm{~d}$ \\
\hline 2831 & $\mathrm{~T}$ & 1.10 & 5.00 & 2 & 2 & 281 & Aborto $115 \mathrm{~d}$ \\
\hline 2822 & $\mathrm{~T}$ & 0.00 & 3.20 & 4 & $4^{*}$ & 270 & Aborto $117,77 \mathrm{~d}$ \\
\hline 5186 & $\mathrm{~T}$ & 2.80 & 4.00 & 3 & 2,3 & 273 & Aborto 129, $95 \mathrm{~d}$ \\
\hline 2709 & $\mathrm{~T}$ & 2.30 & 2.17 & 4 & 1,4 & 274 & Aborto $147,155 \mathrm{~d}$ \\
\hline 2843 & $\mathrm{~T}$ & -1.60 & 3.30 & 3 & $1 *, 3 *$ & 276 & $\begin{array}{l}\text { Aborto 155, 231, } \\
177,124 \mathrm{~d}\end{array}$ \\
\hline 2877 & $\mathrm{~T}$ & 2.20 & 4.00 & 3 & 2,3 & 270 & Aborto $157,174 \mathrm{~d}$ \\
\hline 2807 & $\mathrm{~T}$ & 2.40 & 3.00 & 4 & 1,4 & 276 & Aborto $158,159 \mathrm{~d}$ \\
\hline 5021 & $\mathrm{~T}$ & -0.10 & 4.50 & 3 & 3 & 273 & Aborto $169 \mathrm{~d}$ \\
\hline 5190 & $\mathrm{~T}$ & 1.90 & 1.80 & 3 & $3 *$ & 278 & Aborto $176,154 \mathrm{~d}$ \\
\hline 2800 & $\mathrm{~T}$ & 3.20 & 4.60 & 4 & $1,3^{*}$ & 273 & $\begin{array}{l}\text { Aborto 179, 254, } \\
197 \text { d }\end{array}$ \\
\hline 2943 & $\mathrm{~T}$ & 1.50 & 4.00 & 3 & 2 & 269 & Aborto $185 \mathrm{~d}$ \\
\hline 2744 & $\mathrm{~T}$ & -1.90 & 4.60 & 4 & 4 & 280 & Aborto $197 \mathrm{~d}$ \\
\hline 4886 & $\mathrm{~T}$ & -0.30 & 1.50 & 3 & 2 & 274 & Aborto $216 \mathrm{~d}$ \\
\hline 5100 & $\mathrm{~T}$ & 2.00 & 2.30 & 3 & 3 & 274 & Aborto $220 \mathrm{~d}$ \\
\hline 2880 & $\mathrm{~T}$ & -0.50 & 4.50 & 3 & 2 & 277 & Aborto $51 \mathrm{~d}$ \\
\hline 5206 & $\mathrm{~T}$ & 0.20 & 2.30 & 2 & $2 *$ & 273 & Aborto $53 \mathrm{~d}$ \\
\hline 3299 & $\mathrm{~T}$ & 0.00 & 2.50 & 4 & $2,4^{*}$ & 274 & $\begin{array}{l}\text { Aborto 65, 122, } \\
100 \mathrm{~d}\end{array}$ \\
\hline 2666 & $\mathrm{~T}$ & -0.20 & 4.00 & 4 & 2,3 & 277 & Aborto 79, $69 \mathrm{~d}$ \\
\hline 2797 & $\mathrm{~T}$ & -0.10 & 2.75 & 3 & 3 & 270 & Aborto $86 \mathrm{~d}$ \\
\hline 5115 & $\mathrm{~T}$ & 1.10 & 3.00 & 3 & 2,3 & 270 & Aborto 87, $82 \mathrm{~d}$ \\
\hline 5143 & $\mathrm{~T}$ & 1.40 & 1.50 & 3 & 2 & 276 & Aborto $89 \mathrm{~d}$ \\
\hline 2813 & $\mathrm{~T}$ & -1.70 & 1.80 & 3 & $3 *$ & 278 & Aborto 95, $84 \mathrm{~d}$ \\
\hline 5140 & $\mathrm{~T}$ & 1.70 & 3.30 & 4 & 2 & 273 & Aborto182 d \\
\hline Promedio & & 0.75 & 3.21 & 3.52 & & 275 & 46 abortos \\
\hline
\end{tabular}

* Hubo dos abortos en dicha gestación

T: No es portador para los haplotipos reproductivos 
Cuadro 6. Tasa de concepción del rebaño a través del número de servicios por año, periodo $2000-2016$.

\begin{tabular}{|c|c|c|c|c|c|c|c|c|c|}
\hline \multirow[t]{2}{*}{ Año } & & \multicolumn{6}{|c|}{ Tasa de concepción por número de servicio } & \multicolumn{2}{|c|}{ Vacas (n) } \\
\hline & & 1 & 2 & 3 & 4 & 5 & $6 a+$ & Preñadas & Insemin. \\
\hline \multirow[t]{2}{*}{2000} & $\mathrm{n}$ & 152 & 91 & 59 & 30 & 21 & 18 & 371 & 396 \\
\hline & $\%$ & 38.38 & 22.98 & 14.9 & 7.58 & 5.3 & 4.55 & 93.69 & \\
\hline \multirow[t]{2}{*}{2001} & $\mathrm{n}$ & 137 & 88 & 51 & 19 & 25 & 15 & 335 & 354 \\
\hline & $\%$ & 38.7 & 24.86 & 14.41 & 5.37 & 7.06 & 4.24 & 94.63 & \\
\hline \multirow[t]{2}{*}{2002} & $\mathrm{n}$ & 124 & 71 & 61 & 23 & 22 & 14 & 315 & 332 \\
\hline & $\%$ & 37.35 & 21.39 & 18.37 & 6.93 & 6.63 & 4.22 & 94.88 & \\
\hline \multirow[t]{2}{*}{2003} & $\mathrm{n}$ & 99 & 71 & 38 & 32 & 19 & 17 & 276 & 304 \\
\hline & $\%$ & 32.57 & 23.36 & 12.5 & 10.53 & 6.25 & 5.59 & 90.79 & \\
\hline \multirow[t]{2}{*}{2004} & $\mathrm{n}$ & 130 & 56 & 39 & 19 & 17 & 15 & 276 & 301 \\
\hline & $\%$ & 43.19 & 18.6 & 12.96 & 6.31 & 5.65 & 4.98 & 91.69 & \\
\hline \multirow[t]{2}{*}{2005} & $\mathrm{n}$ & 88 & 66 & 36 & 24 & 12 & 6 & 232 & 243 \\
\hline & $\%$ & 36.21 & 27.16 & 14.81 & 9.88 & 4.94 & 2.47 & 95.47 & \\
\hline \multirow[t]{2}{*}{2006} & $\mathrm{n}$ & 94 & 48 & 39 & 20 & 10 & 16 & 227 & 253 \\
\hline & $\%$ & 37.15 & 18.97 & 15.42 & 7.91 & 3.95 & 6.32 & 89.72 & \\
\hline \multirow[t]{2}{*}{2007} & $\mathrm{n}$ & 106 & 61 & 30 & 24 & 21 & 37 & 279 & 298 \\
\hline & $\%$ & 35.57 & 20.47 & 10.07 & 8.05 & 7.05 & 12.42 & 93.62 & \\
\hline \multirow[t]{2}{*}{2008} & $\mathrm{n}$ & 109 & 79 & 46 & 31 & 21 & 42 & 328 & 344 \\
\hline & $\%$ & 31.69 & 22.97 & 13.37 & 9.01 & 6.1 & 12.21 & 95.35 & \\
\hline \multirow[t]{2}{*}{2009} & $\mathrm{n}$ & 117 & 70 & 47 & 38 & 13 & 43 & 328 & 346 \\
\hline & $\%$ & 33.82 & 20.23 & 13.58 & 10.98 & 3.76 & 12.43 & 94.8 & \\
\hline \multirow[t]{2}{*}{2010} & $\mathrm{n}$ & 123 & 95 & 54 & 36 & 26 & 64 & 398 & 417 \\
\hline & $\%$ & 29.5 & 22.78 & 12.95 & 8.63 & 6.24 & 15.35 & 95.44 & \\
\hline \multirow[t]{2}{*}{2011} & $\mathrm{n}$ & 139 & 97 & 61 & 43 & 19 & 59 & 418 & 435 \\
\hline & $\%$ & 31.95 & 22.3 & 14.02 & 9.89 & 4.37 & 13.56 & 96.09 & \\
\hline \multirow[t]{2}{*}{2012} & $\mathrm{n}$ & 124 & 76 & 53 & 35 & 38 & 127 & 453 & 476 \\
\hline & $\%$ & 26.05 & 15.97 & 11.13 & 7.35 & 7.98 & 26.68 & 95.17 & \\
\hline \multirow[t]{2}{*}{2013} & $\mathrm{n}$ & 120 & 80 & 65 & 46 & 38 & 108 & 457 & 487 \\
\hline & $\%$ & 24.64 & 16.43 & 13.35 & 9.45 & 7.8 & 22.18 & 93.84 & \\
\hline \multirow[t]{2}{*}{2014} & $\mathrm{n}$ & 83 & 65 & 63 & 61 & 40 & 142 & 454 & 541 \\
\hline & $\%$ & 15.34 & 12.01 & 11.65 & 11.28 & 7.39 & 26.25 & 83.92 & \\
\hline \multirow[t]{2}{*}{2015} & $\mathrm{n}$ & 130 & 107 & 78 & 45 & 36 & 155 & 551 & 607 \\
\hline & $\%$ & 21.42 & 17.63 & 12.85 & 7.41 & 5.93 & 25.54 & 90.77 & \\
\hline \multirow[t]{2}{*}{2016} & $\mathrm{n}$ & 50 & 30 & 25 & 19 & 20 & 49 & 193 & 216 \\
\hline & $\%$ & 23.15 & 13.89 & 11.57 & 8.8 & 9.26 & 22.69 & 89.35 & \\
\hline
\end{tabular}


Cuadro 7. Grupos genéticos identificados en una población de vacas Holstein de una ganadería de la costa central del Perú

\begin{tabular}{|c|c|c|c|c|}
\hline Parental común & $\begin{array}{l}\text { Reproductor / } \\
\text { Fundador }\end{array}$ & Progenies con haplotipos & Haplotipo & Generación \\
\hline \multirow{7}{*}{$\begin{array}{l}\text { CAN } 198998 \\
\text { SOVEREIGN }\end{array}$} & \multirow{7}{*}{$\begin{array}{l}\text { USA 040HO02025 } \\
\text { CHIEF, HH1 (0) }\end{array}$} & 007HO00980 MARK & HH1 & 1 \\
\hline & & 071HO00843 LINDY & HH1 & 2 \\
\hline & & 200HO05024 Mr BURNS RC & HH2 & 6 \\
\hline & & 007HO00477 GLENDELL & HH3 & 1 \\
\hline & & 007HO01118 ROTATE & HH3 & 2 \\
\hline & & 029HO05730 MELWOOD & HH3 & 3 \\
\hline & & 007HO03948 EMORY & HH3 & 4 \\
\hline \multirow{9}{*}{$\begin{array}{l}\text { USA } 1491007 \\
\text { 007HO00058 } \\
\text { ELEVATION (0) }\end{array}$} & \multirow{3}{*}{$\begin{array}{l}011 \mathrm{HO} 03562 \\
\text { FORMATION, HH1 } \\
\text { (3) }\end{array}$} & 200HO00044 MORTY ET & HH1 & 4 \\
\hline & & 011HO05284 THRONE & HH1 & 4 \\
\hline & & 011HO05570 FINLEY & HH1 & 4 \\
\hline & $\begin{array}{l}\text { CAN 073HO02479 } \\
\text { OUTSIDE, HH2 (3) }\end{array}$ & 007HO07615 COLBY & $\mathrm{HH} 2$ & 4 \\
\hline & $\begin{array}{l}\text { DEU 198HO00030 } \\
\text { BOSS IRON }\end{array}$ & & HH3 & 3 \\
\hline & USA $007 \mathrm{HO} 06417$ & NLD 388965513 SNOWMAN & HH3 & 5 \\
\hline & $\begin{array}{l}\text { USA 020HO09002 } \\
\text { BESNE BUCK (2) }\end{array}$ & 180HO09612 JOCKO BESNE & HH4 & 3 \\
\hline & $\begin{array}{l}\text { GRB 029HO12209 } \\
\text { SHOTTLE (4) }\end{array}$ & $\begin{array}{l}\text { 151HO00560 ROCKSTAR } \\
\text { RC }\end{array}$ & HH5 & 5 \\
\hline & & 029HO13366 BEACON & HH5 & 5 \\
\hline $\begin{array}{l}\text { USA } 915940 \text { WIS } \\
\text { LEADER }\end{array}$ & $\begin{array}{l}\text { USA 040HO00030 } \\
\text { SKYLINER }\end{array}$ & & HH3 & 2 \\
\hline $\begin{array}{l}\text { USA } 15 \text { HO00040 } \\
\text { IVANHOE SH }\end{array}$ & --- & --- & --- & --- \\
\hline $\begin{array}{l}\text { USA } 1038509 \\
\text { INKA DE KOL }\end{array}$ & --- & --- & --- & --- \\
\hline
\end{tabular}

Tres de los cinco grupos genéticos han presentado, a través de su genealogía, los haplotipos asociados a la reproducción. El primer grupo genético corresponde al toro CHIEF, donde se reporta por primera vez el haplotipo HH1 (VanRaden et al., 2011), el segundo corresponde al toro MARK ANTONY que se reporta el haplotipo $\mathrm{HH}$, el tercero al toro GLENDELL y SKYLER, donde se reporta el haplotipo $\mathrm{HH} 3$ y el cuarto grupo genético al toro SHOTTLE donde se reporta el haplotipo HH5. A través de la progenie de cada grupo genético se introdujo al rebaño los haplotipos reportados en el análisis genómico, determinado mediante el programa de análisis Pedigraph (Garbe y Da, 2008). 


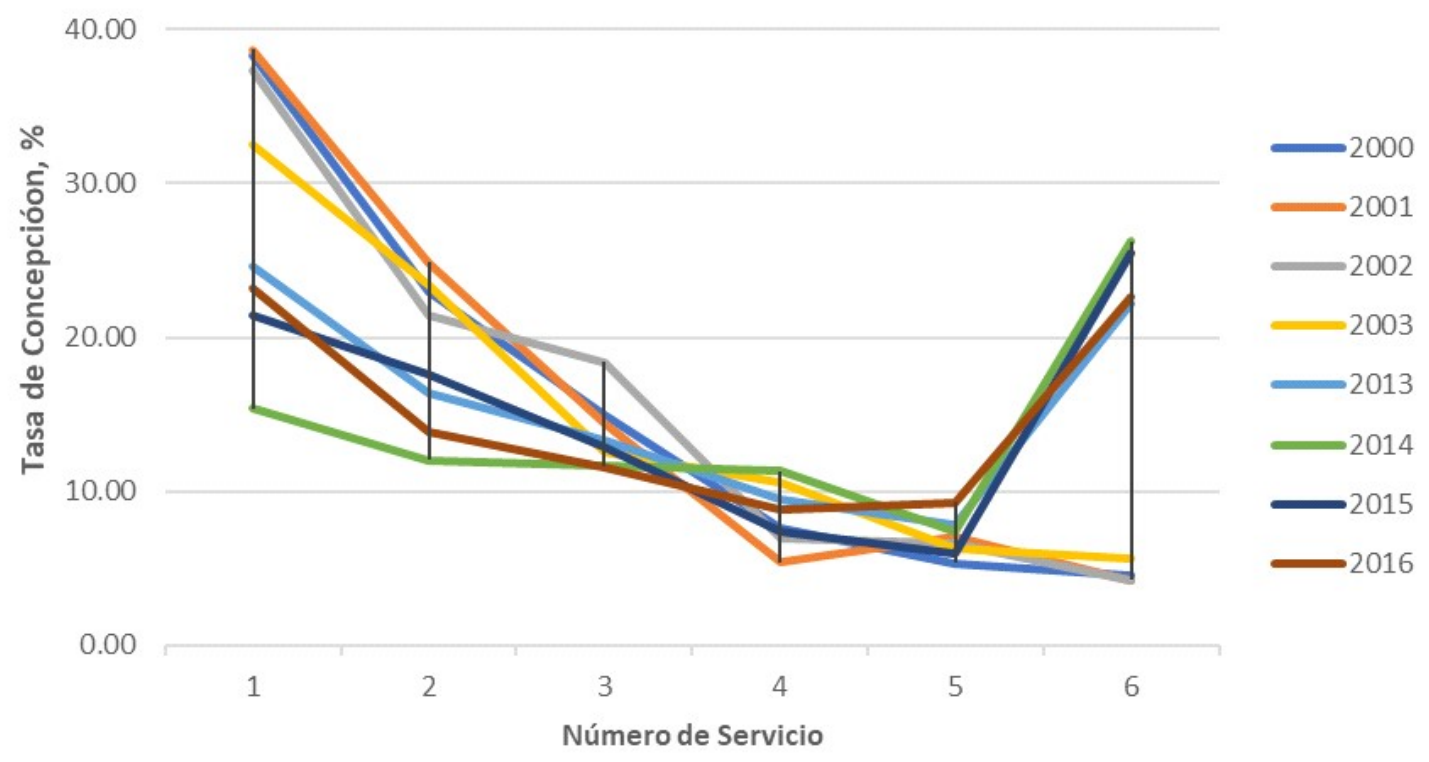

Figura 1. Tasa de concepción de vacas por número de servicio (2000-2004 y 2013-2016)

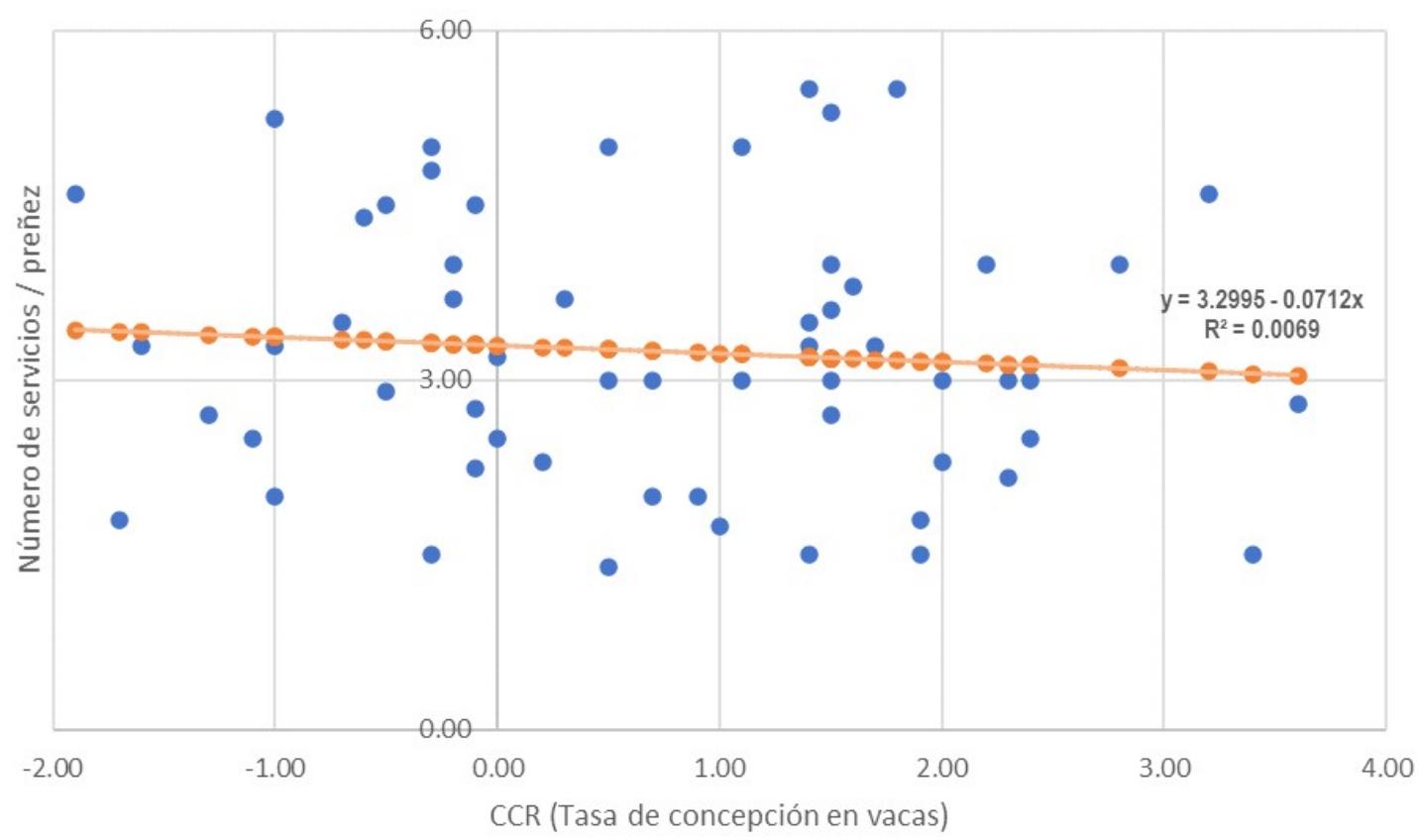

Figura 2. Regresión del número de servicios a la preñez efectiva con los valores de tasa de concepción genómico $(\mathrm{CCR}, \mathrm{n}=58)$ 


\section{ConClusiones}

- La frecuencia de portadores para los haplotipos del tipo $\mathrm{HH} 1$ y $\mathrm{HH} 3$ fue de $5.2 \%$ y para el haplotipo del tipo HH5 de $3.4 \%$.

- El comportamiento reproductivo de las vacas portadoras no fue afectado por la condición de la presencia del haplotipo reproductivo ( $\mathrm{HH} 1, \mathrm{HH} 3$ y $\mathrm{HH} 5)$.

- Los valores genómicos para tasa de concepción en vacas (CCR) no resultó ser un buen indicador del comportamiento reproductivo medido como número de servicios por preñez, siendo no significativa la correlación y la regresión entre las dos características.

- Se determinaron cinco grupos genéticos de padres en el rebaño en estudio y cuatro de ellos presentaron progenie que han sido padres de las vacas y que son portadores para los haplotipos reproductivos.

\section{Agradecimientos}

Al Establo Primavera, por la información de los registros de producción y las muestras para el genotipado de las vacas lecheras. Al Vicerrectorado de Investigación de la Universidad Nacional Agraria La Molina, por el financiamiento para el genotipado de las vacas lecheras, a través de «Innovación Tecnológica - UNALM 2016».

\section{Literatura Citada}

1. CDCB. 2017. Council on Dairy Cattle Breeding. Form GENO. [Internet]. Available in: https://www.uscdeb.com/ what-we-do/genomics

2. Cole JB, VanRaden PM, Null DJ, Hutchison JL, Cooper TA, Hubbard SM. 2014. Haplotype test for recessive disorders that affect fertility and others traits. Beltsville, USA. [Internet]. Available in: https://aipl.arsusda.gov/ reference/recessive_haplotypes_ARRG3.html

3. Cooper TA, Wiggans GR, VanRaden PM, Hutchison JL, Cole JB, Null DJ. 2013. Genomic evaluation of Ayrshire dairy cattle and new haplotypes affecting fertility and stillbirth in Holstein, Brown Swiss and Ayrshire breeds. In: ADSASAS Joint Annual Meeting. Indianapolis, USA

4. Diskin MG, Morris DG. 2008. Embryonic and early foetal losses in cattle and others ruminants. Reprod Dom Anim 43(Suppl 2): 260-267. doi:10.1111/j.14390531.2008.01171.x

5. Diskin MG, Parr MH, Morris DG. 2012. Embryo death in cattle: an update. Reprod Fertil Dev 24: 244-251. doi: 10.1071/RD11914

6. Fritz S, Capitan A, Djari A, Rodriguez $S C$, Barbat A, Baur A,Grohs C, et al. 2013. Detection of haplotypes associated with prenatal death in dairy cattle and identification of deleterious mutations in GART, SHBG and SLC37A2. PLoS ONE 8: e65550. doi: 10.1371/journal.pone. 0065550

7. Garbe JR, Da Y. 2008. Pedigraph: a software tool for the graphing and analysis of large complex pedigree. User manual v. 2.4. Department of Animal Science. University of Minnesota. USA.

8. Gónzalez-Recio O, López de Maturana E, Gutierrez, JP. 2007. Inbreeding depression on female fertility and calving ease in Spanish dairy cattle. J Dairy Sci 90:5744-5752. doi: 10.3168/jds.2007-0203

9. Gutierrez JP, Goyache F. 2005. A note on ENDOG: a computer program for analysing pedigree information. J Anim Breed Genet 122: 172-176. doi: 10.1111/ j.1439-0388.2005.00512.x

10. Holstein Association USA. 2017. [Internet]. Available in: www.holsteinusa.com 
11. Krejcie RV, Morgan DW. 1970. Determining sample sizes for research activities. Educ Psychol Meas 30: 607610. doi: 10.1177/001316447003000308

12. Larkin DM, Daetwyler HD, Hernandez AG, Wright CL, Hetrick LA, Boucek L, Bachman SL, et al. 2012. Whole-genome resequencing of two elite sires for the detection of haplotypes under selection in dairy cattle. P Natl Acad Sci USA 109: 7693-7698. doi: 10.1073/pnas.1114546109

13. Mylrea PJ. 1963. A suspected genetic cause of abortion in cattle. Aust Vet $\mathrm{J}$ 39:35-36. doi: 10.1111/j.1751-0813.1963.tb04174.x

14. Norman HD, Miller RH, Wright JR, Hutchison JL, Olson KM. 2012. Factors associated with frequency of abortions recorded through Dairy Herd Improvement test plan. J Dairy Sci 95: 4074-4084. doi: 10.3168/jds.2011-4998.

15. Parland SMc, Kearney JF, Rath M, Berry DP. 2007. Inbreeding effects on milk production, calving performance, fertility, and conformation in Irish Holstein-Friesians. J Dairy Sci 90: 44114419. doi: 10.3168/jds.2007-0227
16. USDA APHIS. 2017. United State Department of Agriculture. Animal and Plant Health Inspection Services. [Internet]. Available in: www.aphis.usda.gov/aphis

17. VanRaden PM, Sanders AH, Tooker ME, Muller RH, Norman HD, Kuhn MT, Wiggans GR. 2004. Development of a national genetic evaluation for cow fertility. J Dairy Sci 87: 2285-2292. doi: 10.3168/jds.S0022-0302(04)70049-1

18. VanRaden PM, Olson KM, Null DJ, Hutchison JL. 2011. Harmful recessive effects on fertility detected by absence of homozygous haplotypes. J Dairy Sci 94: 6153-6161. doi: $10.3168 /$ jds.20114624

19. VanRaden PM, Null DJ, Sonstegard TS, Adams HA, Van Tassell CP, Olson KM. 2012. Fine mapping and discovery of recessive mutations that cause abortions in dairy cattle. J Dairy Sci 95(Supp1 2): Abstract.

20. Wijeratne WVS, Stewart DL. 1971. Population study of abortion in cattle with special reference to genetic factors. Anim Prod 13: 229-235. doi: 10.1017/ S0003356100029664 